\title{
Linfadenitis causada por Mycobacterium malmoense en paciente infectado con virus de inmunodeficiencia humana
}

\author{
LILIAN M. MEDEROS, DANIEL GONZÁLEZ, DANIEL PÉREZ, \\ ALINA PANEQUE y ERNESTO MONTORO
}

\section{Lymphadenitis caused by Mycobacterium malmoense in HIV infected patient}

We report the first Cuban case of mycobacteriosis caused by Mycobacterium malmoense in an HIV infected patient who developed submandibular ulcerative adenopathies. Culture of clinical samples yielded a non-pigmented, slowly growing mycobacteria strain that was classified in the Runyon Group I. The identification was complemented by biochemical tests and analysis of mycolic acid fractions.

Key words: Lymphadenitis, Mycobacterium malmoense, HIV infected patient, mycolic acids.

Palabras claves: Linfadenitis, Mycobacterium malmoense, Paciente infectado por VIH, Ácidos micólicos.

\section{Introducción}

Dentro de la micobacteriología, Mycobacterium tuberculosis sigue siendo el agente causal más importante de infecciones tanto pulmonares como extrapulmonares; sin embargo, existen además enfermedades producidas por otras especies de micobacterias (no tuberculosas) dignas de ser tenidas en cuenta ${ }^{1,2}$.

Con el nombre de "micobacteriosis atípicas" se designa globalmente a todas las infecciones causadas por micobacterias, excepto las producidas por $M$. tuberculosis y Mycobacterium leprae. Las enfermedades producidas por ellas alcanzan grados significativos y son un importante problema de salud, tanto en los países en desarrollo con altas tasas de incidencias y pocos recursos, como en los países desarrollados, donde el virus de inmunodeficiencia humana (VIH) y la migración han complicado grandemente esta situación. Estas micobacterias son usualmente saprófitas para el ser humano y se encuentran distribuidas en el medio ambiente; no se consideran patógenas estrictas como es el caso de $M$. tuberculosis y sólo en determinadas circunstancias, como son los pacientes inmunocomprometidos, pueden actuar como patógenas y diseminarse $\mathrm{e}^{1-3}$.

Las micobacteriosis atípicas más frecuentes en humanos y en pacientes infectados por VIH son las producidas por el complejo Mycobacterium avium (MAC) ${ }^{4-7}$. Mycobacterium kansasii es otra de las especies aisladas de estos pacientes como causa de infecciones pulmonares y diseminadas ${ }^{3}$. Especies saprófitas como Mycobacterium gordonae se han manifestado también como patógenos oportunistas en este grupo de riesgo ${ }^{8-10}$.

Describimos un caso de micobacteriosis atípica causada por Mycobacterium malmoense, en un paciente infectado por VIH y adenopatías ulcerativas submandibulares.

\section{Caso Clínico}

Paciente sexo masculino con 25 años de edad, portador de infección por VIH detectada en 1997 , actualmente con SIDA. Entre 2000 y 2002 presentó varios cuadros de fiebre, acompañados de

Instituto de Medicina Tropical Pedro Kourí, La Habana, Cuba.

Laboratorio Nacional de Referencia e Investigaciones de Micobacterias y Tuberculosis. Centro Colaborador OPS/OMS.

Recibido: 24 octubre 2003

Aceptado: 22 junio 2004 
tos con escasa expectoración, que mejoraban con tratamiento antimicrobiano, y también comenzó a experimentar considerable pérdida de peso.

En agosto del 2002 le inició tratamiento antiretroviral con zidovudina, lamivudina e indinavir por CD4 en 5\% (51 células $/ \mathrm{mm}^{3}$ ) y carga viral de 720.000 copias/ml de sangre. En octubre del 2002 nuevamente fue hospitalizado, presentando adenomegalia submandibular que evolucionó hacia la necrosis y ulceración, no mejorando con tratamiento antimicrobiano ni drenaje quirúrgico; incluso el compromiso ganglionar se propagó a la región lateral del cuello y supraclavicular derecha, con adenopatías de tamaño variable y consistencia dura, algunas ulceradas y con secreción purulenta. La VHS era de $133 \mathrm{~mm} / \mathrm{h}$.

Se tomó biopsia de las diferentes lesiones, enviándose a cultivo bacteriológico corriente. Para el estudio de micobacterias se descontaminó el material de estudio obtenido a partir de biopsias con $\mathrm{H}_{2} \mathrm{SO}_{4}$ al $4 \%$, según técnicas recomendadas para el aislamiento y cultivo micobacteriano ${ }^{11}$. Luego fueron cultivadas en medio Lowenstein Jensen e incubadas a $37^{\circ} \mathrm{C}$. Las lecturas del cultivo se realizaron cada 7 días; a las 3-4 semanas de incubación se encontró el aislamiento de colonias de crecimiento lento, sin pigmento, de bordes irregulares, en todos los cultivos. El recuento de colonias osciló entre 5 y 7 por placa. A partir de las colonias se realizó la tinción de ZiehlNeelsen para detectar la presencia de bacilos/ ácido/alcohol/resistentes (BAAR) con resultado positivo.

Para el estudio de clasificación micobacteriana se utilizó las pruebas recomendadas por protocolo de trabajo elaborado por INCO-PRA ${ }^{12}$ : crecimiento a $25^{\circ} \mathrm{C}, 45^{\circ} \mathrm{C}$ y $37^{\circ} \mathrm{C}$, producción de pigmento, crecimiento en agar nutriente, y las siguientes pruebas bioquímicas: $\mathrm{TCH}, \mathrm{NaCl} 5 \%$, $\mathrm{PNB}$, ác pícrico, hidroxilamina, isoniacida, catalasa semicuantitativa, nitratasa, ureasa, pirazidamidasa, fosfatasa ácida, arilsulfatasa (3-14 días), $\beta$-galactosidasa, niacina, tween 80 (5-10 días) y telurito de potasio.

Como técnica diagnóstica de confirmación se utilizó el estudio de las fracciones de ácidos micólicos por la técnica de cromatografía en capa delgada bidimensional, según técnica descrita y recomendada en la literatura médica ${ }^{13-16}$.

A través del estudio bioquímico la cepa fue identificada como M. malmoense, y fue confirmada mediante la técnica alternativa diagnóstica de cromatografía en capa delgada bidimensional, para el análisis del patrón de las fracciones de ácidos micólicos. El patrón descrito fue: alfa, alfa' y cetomicolato, además de la mancha que caracteriza el resto de los ésteres metílicos de los ácidos grasos.

Tras los resultados microbiológicos obtenidos se inició tratamiento con rifabutina, claritromicina y etambutol durante 6 semanas. Las lesiones fueron mejorando hasta su desaparición; simultáneamente el recuento de CD4 subió hasta 168 células $/ \mathrm{mm}^{3}$ y la VHS regresó a cifras normales.

\section{Discusión}

En la literatura han sido varios los reportes de micobacteriosis producidas por M. malmoense, a pesar de que esta especie no es común encontrarla. Casi siempre se ha descrito asociada a pacientes con algún tipo de inmunocompromiso, principalmente produciendo infecciones pulmonares en adultos. Uno de estos estudios describe las diversas infecciones asociadas a esta especie, en diferentes estados de E.U.A. ${ }^{17,18}$; la mayoría de los casos M. malmoense en niños ha sido vinculada a enfermedades pulmonares y en especial a adenitis ${ }^{17-20,21}$. También ha sido asociada a micobacteriosis diseminada en pacientes infectados por VIH, en algunos casos causando su muerte ${ }^{19,21,23,24}$, y como caso inusual esta especie micobacteriana se aisló en un paciente que padecía de un pseudotumor en pulmón, y también como agente causal en un caso de tenosinovitis ${ }^{25,26}$.

Creemos que la fusión de los métodos bioquímicos convencionales para la identificación micobacteriana, con los métodos alternativos diagnósticos como son los estudios de las fracciones de ácidos micólicos micobacterianos por cromatografía en capa delgada, podrá otorgar un mayor beneficio diagnóstico.

Este estudio constituye el primer reporte de M. malmoense en un paciente portador de VIH en Cuba y el primer caso de linfadenitis por micobacterias no tuberculosas en este grupo de riesgo.

Se recomienda para el grupo de pacientes inmunocomprometidos por diferentes causas, poner especial interés a los aislamientos de micobacterias, incluyendo aquellas especies a las que generalmente no se les atribuye interés clínico, pues las consecuencias encontradas en este grupo de riesgo son complejas.

\section{Resumen}

Se reporta el primer caso cubano de micobacteriosis causada por Mycobacterium malmoense en un paciente infectado por el virus de inmunode- 
ficiencia humana (VIH), el cual presentaba adenopatías ulcerativas submandibulares. A partir de las muestras tomadas de las lesiones ulceradas, se aisló una cepa de micobacteria no pigmentada de crecimiento lento, perteneciente al grupo I de Runyon, posteriormente fue clasificada por test bioquímico y por el análisis de las fracciones de ácidos micólicos, como Mycobacterium malmoense.

\section{Bibliografía}

1.- Sauret J, Hernándex-Flix S. Tratamiento actual de las micobacteriosis. Med Clin (Barc) 1990; 95: 64-6.

2.- Casal M. Micobacteriología. Diagnóstico y Salud Pública. Segunda Edición, editado por el Departamento de Higiene y Enfermedades Tropicales, Madrid, España. 1991.

3.- Sullivan A K, Hannan M M, Azadian B S, Georges K L. Acid-alcohol fase bacilli in sputa of HIV-infected patients. Int J STD AIDS 1999; 10 (9): 606-8.

4.- Casal M. La aviumosis como infección emergente y su quimioterapia. Rev Esp Quimioter 1997; 10 (2): 131-7.

5.- Brodt H R, Enzensberger B R, Kamps B S, Keul H G, Helm E B. Impact of disseminated Mycobacterium avium complex infection on survival of HIV infected patients. Eur J Med Res 1997; 2: 106-10.

6.- Richter E, Wessling J, Lugering N, Domschker W, Rusch-Gerdes S. Mycobacterium avium subsp paratuberculosis in patient with HIV, Germany. Emerg Infect Dis 2002; 8 (7): 123-8.

7.- Mederos L M, Rodríguez F, Blanco F, Cabrera J, Echemendía M, Montoro E. Reporte de Mycobacterium avium-intracellulare asociado a micobacteriosis renal. Rev Cub Med Trop 2003; 55 (1): 58-60.

8.- Saggese D, Compadretti G C, Burnelli R. Nontuberculous mycobacterial adenitis in children: Diagnostic and therapeutic management. Am J Otolaryngol 2003; 24 (2): 79-84.

9.- Rusconi S, Gori A Vago L, Marchetti G, Franzetti F. Cutaneous infection caused by Mycobacterium gordonae in immunodeficiency virus-infected patient receiving antimycobacterial treatment [letter]. Clin Infect Dis 1997; 25 (6): 1490.

10.- Mederos L M, Ruíz A, Valero-Guillén P L. Micobacteriosis en paciente VIH+. Rev Hospital Nacional Baldomero Sommer 2000; 3 (1): 26-8.

11.- Casal M. Microbiología clínica de las enfermedades por micobacterias (Tuberculosis, Lepra y Micobacteriosis). 1990, Facultad de Medicina, Universidad de Córdova, España.

12.- Cardoso, S. Biochemical identification protocol INCO-
PRA, 2003.

13.- Minnikin D E, Minnikin S M, Parlett J H, Goodfellow M. Mycolic acid patterns of some rapidly-growing species of Mycobacterium. Zbl Bakt Hyg 1985; A25960.

14.- Valero-Guillén P L, Martín-Luengo F. Cromatografía en capa delgada y cromatografía de gases en la identificación de micobacterias de interés clínico. Enf Infec Microbiol Clin 1987; 5 (6): 67-72.

15.- Leite C Q F, Souza C W O, Leite S R A. Identification of Mycobacteria by thin layer chromatographic analysis of mycolic acids and conventional biochemical method: Four years of experience. Mem Inst Oswaldo Cruz, Nov/Dec 1998; 93 (6): 801-5.

16.- Walkiewicz R, Saflanowska A, Grubek-Jaworska H, Zalewska-Schonthaler N, Glapinski J, Chazan R. Use of mycolic acid analysis in diagnosis of tuberculosis and mycobacteriosis- three-year experience. Pneumonol Alergol Pol 2002; 70 (9-10): 444-9.

17.- Buchholz U T, McNell M M, Keyes L E, Good R C. Mycobacterium malmoense infections in the United States, January 1993 through June 1995. Clin Infect Dis 1998; 27 (3): 551-8.

18.- [No authors listed]. Pulmonary disease caused by Mycobacterium malmoense in HIV negative patients: 5-yr follow-up of patients receiving standardized treatment. Eur Respir J 2003; 21 (3): 478-82.

19.- Willocks L, Leen C L, Brettle RP, Bhumbra N. Isolation of Mycobacterium malmoense from HIVpositive patients. J Infect 1993; 26 (3): 345-6.

20.- Claydon E J, Coker R J, Hazrris J R. Mycobacterium malmoense infection in HIV positive patients. J Infect 1991; 23 (2): 191-4.

21.- Elliott B A, Griffith D E, Wallace R J Jr. Diagnosis of nontuberculous mycobacterial infection. Clin Lab Med 2002; 22 (4): 911-25.

22.- Enzensberger R, Hunfeld K P, Krause M, Rusch-Gerdes $\mathrm{S}$, Brade V, Boddinghaus B. Mycobacterium malmoense infections in immunocompetent patients. Eur J Clin Microbiol Infect Dis 1999; 18 (8): 579-81.

23.- Chocarra A, González-López A, Breznes M F, Ricart A. Disseminated infection due to Mycobacterium malmoense in a patient infected with human immunodeficiency virus. Clin Infect Dis 1994; 19 (1): 203-4.

24.- Fakih M, Chapalamadugu S, Ricart A, Corriere N, Amsterdam D. Mycobacterium malmoense bacteremia in two AIDS patients. J Clin Microbiol 1996; 34 (3): 731-3.

25.- Yohanathan K, Elliott M W, Moxham J, Good R C. Pseudotumor of the lung caused by Mycobacterium malmoense infection in an HIV positive patient. Thorax 1994; 49 (2): 179-80.

26.- Syed A, O'Flanagan J. Mycobacterium malmoense infection. An unusual cause of wrist swelling. J Hand Surg [Br] 1998; 23 (6): 811-2.

Correspondencia a:

Lilian M. Mederos Cuervo

E-mail: mederos@ipk.sld.cu 